\title{
Application of Spontaneous Photon Emission in the Growth Ages and Varieties Screening of Fresh Chinese Herbal Medicines
}

\author{
Xiaolei Zhao, ${ }^{1}$ Jingxiang Pang, ${ }^{1,2}$ Jialei Fu, ${ }^{3}$ Meina Yang, ${ }_{1,}^{1}$ Eduard Van Wijk, \\ Yanli Liu, ${ }^{3}$ Hua Fan, ${ }^{3}$ Yufeng Zhang, ${ }^{2}$ and Jinxiang Han ${ }^{1,2}$ \\ ${ }^{1}$ Department of Biochemistry and Molecular Biology, Shandong University, Jinan 250012, China \\ ${ }^{2}$ Shandong Medicinal Biotechnology Center, Key Laboratory for Biotech-Drugs of The Ministry of Health, \\ Shandong Academy of Medical Sciences, Jinan 250062, China \\ ${ }^{3}$ Shandong Academy of Traditional Chinese Medicine, Jinan 250355, China \\ ${ }^{4}$ Sino-Dutch Centre for Preventive and Personalized Medicine/Centre for Photonics of Living Systems, \\ Leiden University, Leiden, Netherlands \\ ${ }^{5}$ Meluna Research, Geldermalsen, Netherlands \\ Correspondence should be addressed to Jinxiang Han; samsjxhyx@163.com
}

Received 19 July 2016; Revised 23 November 2016; Accepted 27 December 2016; Published 31 January 2017

Academic Editor: Cheorl-Ho Kim

Copyright (c) 2017 Xiaolei Zhao et al. This is an open access article distributed under the Creative Commons Attribution License, which permits unrestricted use, distribution, and reproduction in any medium, provided the original work is properly cited.

\begin{abstract}
Ultraweak photon emission emitted by all living organisms has been confirmed to be a noninvasive indicator for their physiological and pathological characteristics. In this study, we investigated the characteristics of spontaneous photon emission (SPE) and the contents of specific active compounds of roots and flowers buds of several fresh Chinese herbal medicines (natural medicines) with different growth ages and varieties. The results revealed that the contents of specific active compounds from same species herbs with different growth ages and varieties were significantly different, and this difference could be reflected by their SPE. Because the contents of specific bioactive constituents in Chinese herbs are closely related to their quality and curative effect, the SPE measurement technique may contribute to the quality control of Chinese herbal medicine in the future.
\end{abstract}

\section{Introduction}

All living organisms including animals, plants, algae, and bacterium spontaneously generate ultraweak photon emission (UPE), which is often called biophoton [1]. The emission ranges from the ultraviolet to near-infrared with intensities from tens to thousands photons per square centimeter per second [2]. The origin of this light energy is related to the chemiexcitation of biological molecules during oxidative metabolic processes $[3,4]$. Therefore, the biophoton is closely related to various types of factors, such as exogenous toxins [5-7], pathogen attack $[8,9]$, temperature $[10,11]$, and acute stress [12-16], and it can reflect the physiological and pathological conditions of cells and tissues in a whole view. As a result, the measure of biophoton emission as a novel and noninvasive method has attracted considerable attention for monitoring the state of living organism and has been used in many fields, such as assessing cancer [17-20] and other health problems [21-26], detecting the quality of food [27], and the application in agriculture [7, 13].

Chinese herbal medicine (CHM; also known as Chinese herbs or Chinese materia medica), having a history of thousands of years, recently became widespread throughout the world due to their potential as an alternative complementary therapy and a source for discovering new drugs [28-30]. It possesses a holistic health care concept and regards disease as the imbalance of energy. Additionally, CHM adheres to the philosophy of cure instead of treatment [31]. The accumulated knowledge about CHMs makes their use safe and reasonable. However, the good and bad qualities of herbs have been intermingled in the market for the past few decades, which seriously affects the curative effect of Chinese medicinal formulae and the development of CHM. Therefore, how to discriminate the good qualities of herbs from bad ones 


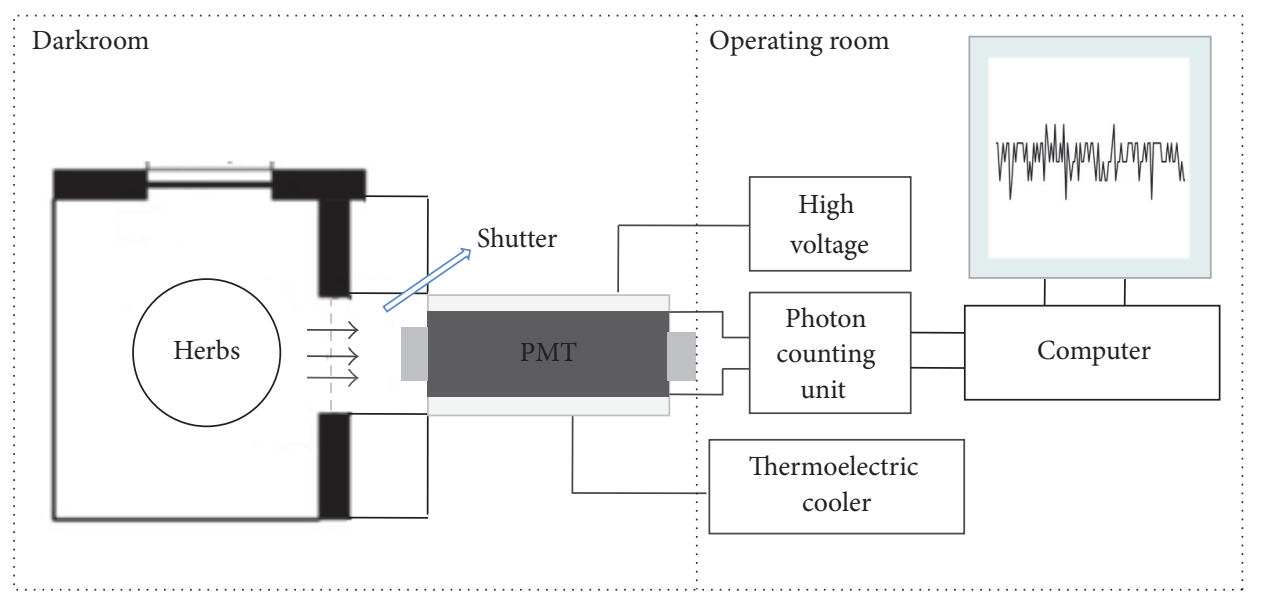

FIGURE 1: The schematic diagram of biophoton detection system used in this study.

becomes a thorny problem in the quality control of CHM [32]. There are many factors affecting the quality of herbs including growth ages, varieties, harvest season, processing, and storage [33]. However, it is hard to identify the varieties and growth ages and so forth of herbs only through their appearances, especially those of root herbs. It will seriously affect the quality of herbs from the source. In order to standardize the quality of CHM to some extent, a document named "General Guidelines for Methodologies on Research and Evaluation of Traditional Medicines" was enacted by World Health Organization in 2000 [34].

Since the active constituent(s) of the vast majority of herbs are often unknown, it is still inadequate to check the quality of herbs based on analyzing the constituents of them despite the fact that great efforts have been made in the quality control of CHM so far $[31,35,36]$. Therefore, the methods for quality control of $\mathrm{CHM}$ have not been recognized by the scientific communities currently. In order to discriminate the quality and identity of CHM meaningfully and properly, the new method retaining the traditional aspect of CHM needs to be employed. As the UPE reflects biological change in the holistic levels, which is consistent with the reflection of the CHM, the measurement of UPE is considered to be a promising means for the analysis of Chinese herbs. Recent studies have reported that DL were correlated significantly with the bioactive chemical constituents and can be used to predict and assess the Chinese herb's therapeutic properties [37, 38]. Taking into account the important roles of growth ages and varieties of herbs in their quality and the fundamental role of procurement quality of fresh herbs in the quality of CHM, additionally, verifying whether the spontaneous photon emission (SPE) could be used as a novel and noninvasive tool for the quality screening of fresh CHM, we carried out an investigation in the characteristics of SPE from the roots and flowers buds of several fresh CHMs considering their growth ages and varieties with the use of a sensitive photomultiplier tube (PMT) in this paper. And the contents of specific active compounds of these herbs were also analyzed, which could reflect the quality of herbs.
The results displayed that the contents of specific active compounds from same species herbs with different growth ages and varieties were significantly different, and this difference could be reflected by their SPE. These results indicated that this novel method may play a critical role in the quality screening of fresh CHMs in the future or at least in the screening of herbs with different growth ages and varieties

\section{Materials and Methods}

\subsection{Spontaneous Photon Emission (SPE) Measurements}

2.1.1. SPE-Detection System. Schematic diagram of the SPEdetection system used in our experimental study is shown in Figure 1. The components of this system include a dark chamber, a photomultiplier tube (PMT), a photon counting unit, a high voltage supply, a thermoelectric cooler, and a computer with photon count data software. The core components of the detection system are PMT R375 and photon counting unit C9744 (Hamamatsu Photonics K.K., Iwata, Japan). In front of the PMT is a shutter whose role is to avoid the disturbance of external light to the PMT.

The numbers of emitted photons from the samples were detected by the low noise and highly sensitive PMT working in single photon counting mode with a spectral response ranging from 160 to $850 \mathrm{~nm}$ and wavelength of maximum response was $420 \mathrm{~nm}$. Subsequently, the photons were processed by the photon counting unit and fed into the computer which displayed the intensity in counts per $100 \mathrm{~ms}$. To decrease the dark current ( $5 \mathrm{nA})$ and increase the sensitivity, the PMT was cooled down to $-30^{\circ} \mathrm{C}$ using thermoelectric cooler C10372 (Hamamatsu Photonics K.K.), and the window of PMT was shielded by a dark chamber which was used to ensure light shielding and magnetic shielding. And then the PMT and the dark chamber were all placed in a dark room in order to eliminate the interference of external light further. In the dark chamber, a sample holder was set up not only to fasten the position of samples but also to ensure that the distance $(3 \mathrm{~cm})$ between the samples and the window of 


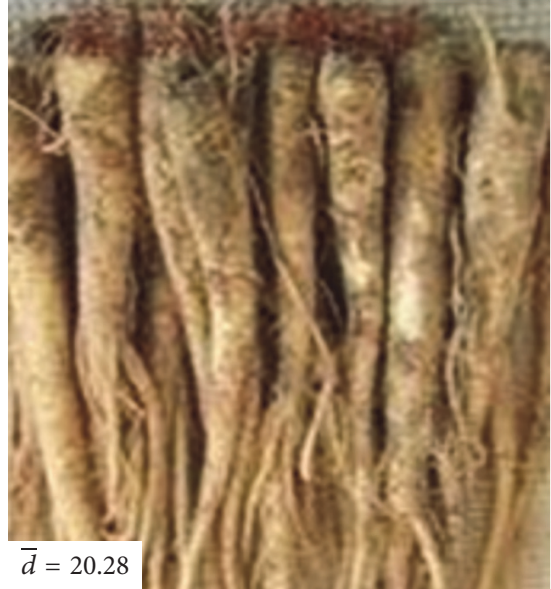

(a)

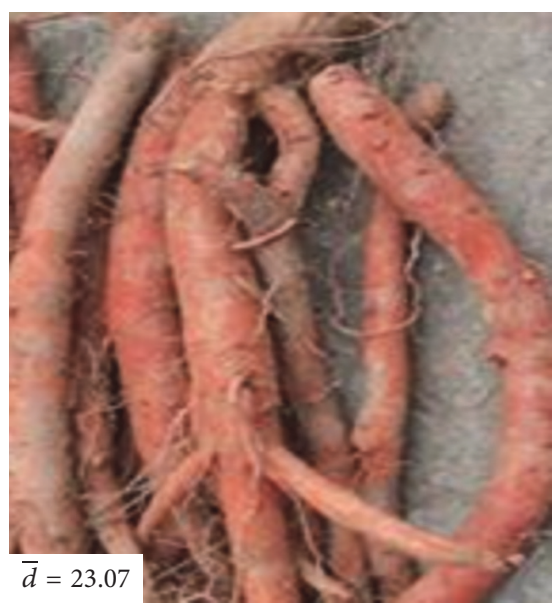

(c)

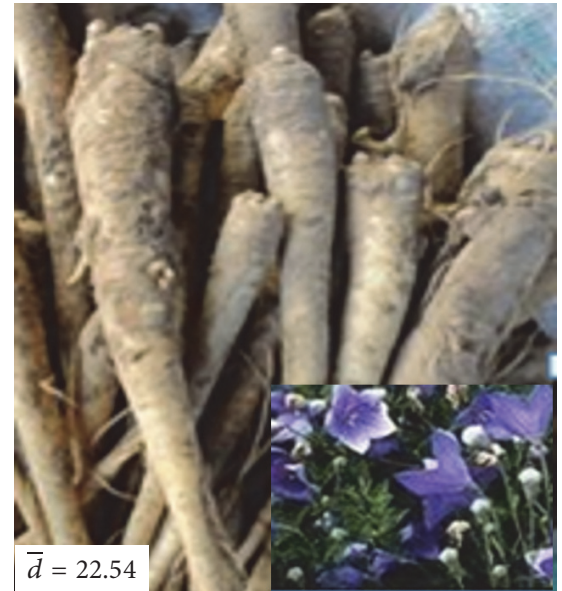

(b)

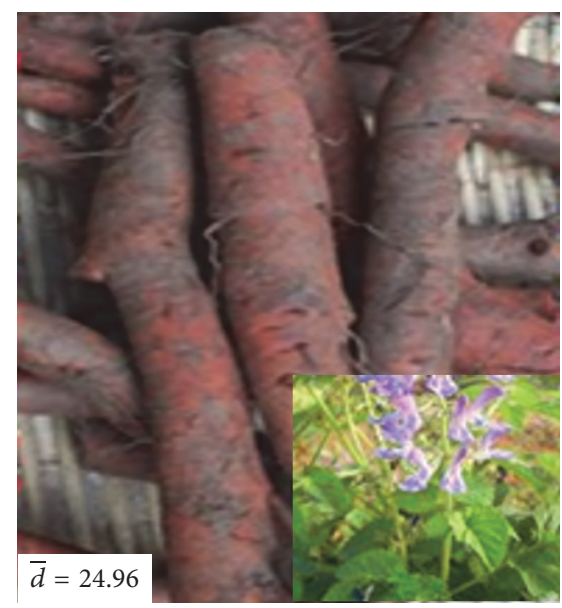

(d)

FIGURE 2: The roots of different growth ages of purple flower P. grandiflorum and purple flower S. miltiorrhiza in this study. (a) One-year-old purple flower P. grandiflorum; (b) two-year-old purple flower P. grandiflorum; (c) one-year-old purple flower S. miltiorrhiza; (d) two-year-old purple flower S. miltiorrhiza. $\bar{d}$ means the average diameter of measured samples.

the PMT is same for all the measurement. Except for the PMT and the dark chamber, the other components of the detection system were placed in the operating room. During the period of measurement, temperature in the dark room was controlled at $25 \pm 1^{\circ} \mathrm{C}$ by the temperature controller.

2.1.2. Samples and Preparation. P. grandiflorum, S. miltiorrhiza, and Lonicera japonica (L. japonica) are planted in large areas in Shandong province. However, the growth ages and varieties of them are very confusing and they often mix together in the market. Unfortunately, some studies suggested that the growth ages and varieties of $P$. grandiflorum and S. miltiorrhiza seriously affected their active constituents, quality, and curative effect [35,39-41]. Besides, there are more varieties of $L$. japonica in the market, which result in the error of clinical use of them and not reaching the curative effect that we expect [42]. Thus, the roots of different varieties of P. grandiflorum (white flower P. grandiflorum and purple flower P. grandiflorum) and S. miltiorrhiza (white flower S. miltiorrhiza and purple flower S. miltiorrhiza) with different ages (one-year-old and two-year-old) and the flowers buds of L. japonica (four seasons of L. japonica and Jiufeng number 1 ) were collected, respectively, as the experimental samples in this study. The photo samples were shown in Figures 2 and 3. Each kind of root samples with same growth age contained 30 samples collected from the same part of 30 plants in the similar growth states for 30 independent experiments in order to eliminate the effects by chance. And each variety of flower samples contained 120 flowers buds and was randomly divided into 20 portions (each portion includes 6 flowers buds) for 20 independent experiments. All of the samples were collected from the plantation of Shandong University of Traditional Chinese Medicine within about $1.5 \mathrm{~h}$. At about 8:30 in everyday morning from 12 August to 30 August, the samples were collected. When the samples were picked, they were put in fresh-keeping bags and then preserved in a fresh-keeping box. Before measurement, each sample was pretreated as follows. For the roots of $P$. grandiflorum and 


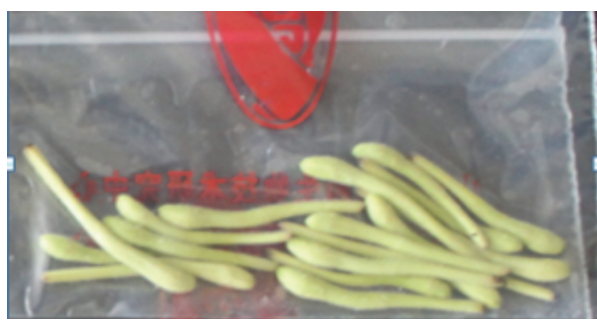

(a)

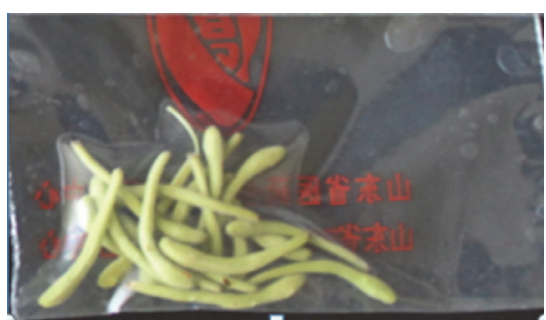

(b)

FIgure 3: The flowers buds of different varieties of L. japonica used in this study. (a) Four seasons of L. japonica; (b) Jiufeng number 1.

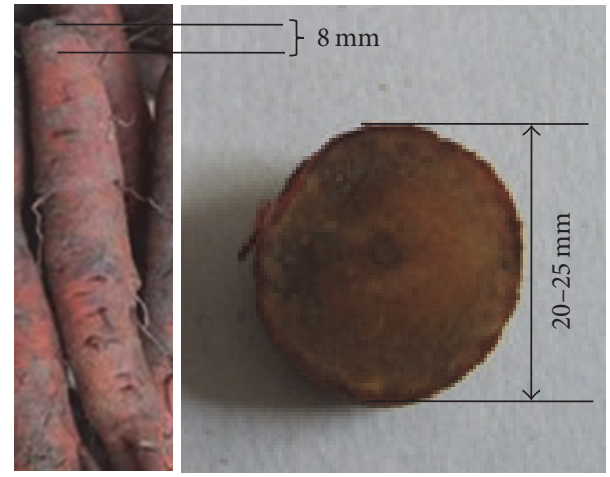

Figure 4: The diagram of slice sample of S. miltiorrhiza. The slice was cut from the top of the root and the thickness of all the slices was $8 \mathrm{~mm}$. The diameter of all the slices ranges from $20 \mathrm{~mm}$ to $25 \mathrm{~mm}$.

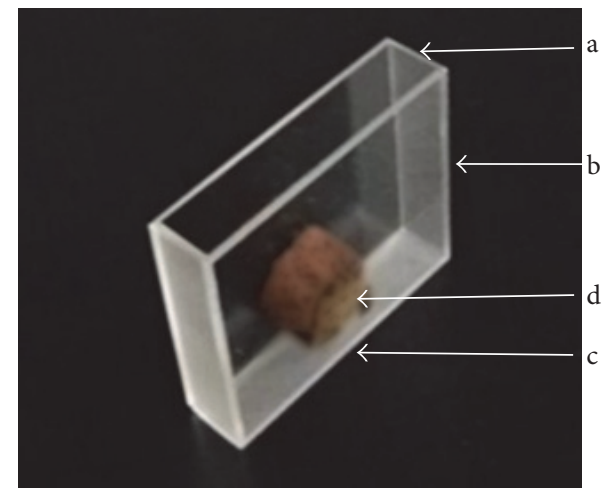

FIGURE 5: The diagram of cuvette used in this study. (a) The inside width of the cuvette was $1 \mathrm{~cm}$. (b) The inside height of the cuvette was $4 \mathrm{~cm}$. (c) The inside length of the cuvette was $5 \mathrm{~cm}$. (d) The samples were placed in the middle of the bottom of the cuvette.

S. miltiorrhiza, each sample was cleaned, one piece was cut from the top of each sample (the diagram of slice sample was displayed in Figure 4), and then the slices were put into clean quartz cuvettes (as shown in Figure 5(d)); for the flowers buds of $L$. japonica, each sample was cleaned and every 6 flowers buds as one portion were put into the clean quartz cuvette. Subsequently, their exposed areas represented by $S$ to the PMT side were measured. In order to eliminate the influence of external light, the pretreated samples were placed in a completely dark room with controlled humidity (50\%) and temperature $\left(25 \pm 1^{\circ} \mathrm{C}\right)$ for $0.5 \mathrm{~h}$ before measurement to eliminate delayed luminescence.

2.1.3. Measurement Procedures. Each test always commenced at the same time of day (10:30 a.m.) in order to try to keep all of the measurement conditions consistent. The procedures for measuring SPE were as follows. The background emission (BG) of the blank cuvette without any sample was measured for $3 \mathrm{~min}$. Then, the shutter was closed and the processed samples were put inside the dark chamber on the sample holder of the detection system under the condition of complete darkness within $30 \mathrm{~s}$. After that, the shutter was opened and the SPEs of the samples were recorded. We sequentially measured 10 samples each day and each sample was measured 3 times. In order to shorten the total measurement time, to keep the samples fresh, we set the duration of each measurement for $5 \mathrm{~min}$. In the whole measurement process, the time interval $(\Delta t)$ was $100 \mathrm{~ms}$. The obtained data were finally corrected by the corresponding background emissions.

2.2. Determination of Specific Bioactive Constituents of Chinese Herbs. The contents of specific bioactive constituents in Chinese herbs are closely related to their quality and curative effect. Therefore, we sent our herbal samples to the Shandong Institute for Food and Drug Control for detecting the specific bioactive constituents in order to analyze whether the contents of specific active compounds of herbs with different growth ages and varieties were different. Total saponins in P. grandiflorum, tanshinone IIA, and salvianolic acid B in S. miltiorrhiza, galuteolin, and chlorogenic acid in $L$. japonica were detected, respectively, according to the Chinese Pharmacopoeia and relevant literature.

2.3. Data Analysis. All of the data of SPEs from each sample were normalized by their exposed areas. And the statistical analysis of photon count data of samples was presented in mean \pm SD (standard deviation) which was performed with SPSS 18.0 software. The data graph was made with program Origin 8.0. The paired $t$-test was used to compare the data from the samples with two different growth ages and varieties. In all statistical tests, $P \leq 0.05$ was considered significant. 


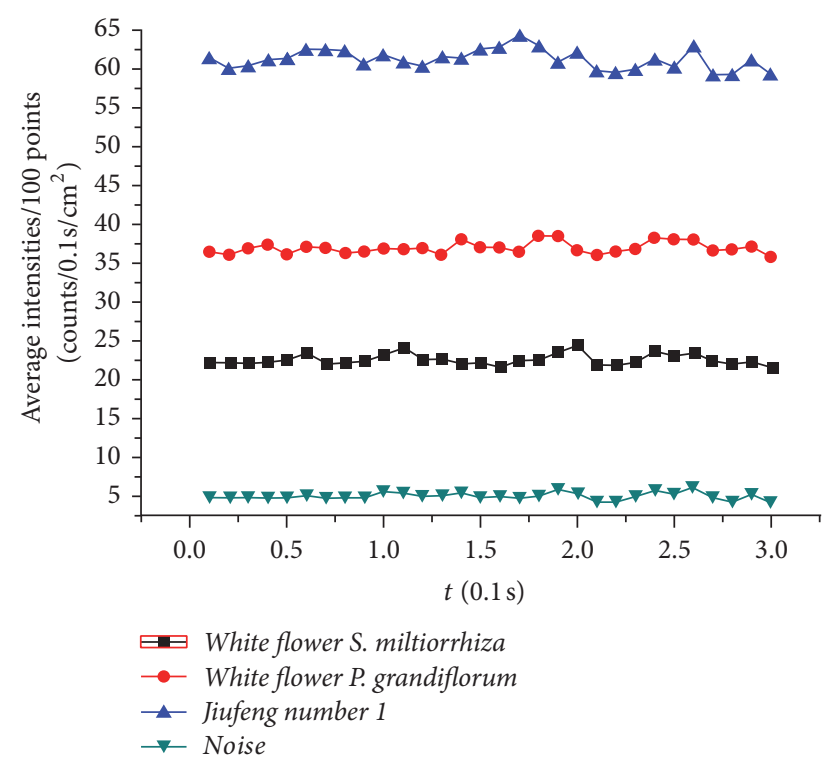

FIGURE 6: The average intensity of every 100 points of SPE from the roots of white flower P. grandiflorum (red dot) and white flower S. miltiorrhiza (black square) with two years of age as well as the $L$. japonica's flowers buds of Jiufeng number 1 (blue equilateral triangle) and the background noise (dark green inverted triangle).

\section{Results}

3.1. The Differences of SPE Intensity and Contents of Specific Bioactive Constituents between the Samples Differing in Growth Age. The SPE and contents of specific bioactive constituents of roots of white flower P. grandiflorum, purple flower P. grandiflorum, white flower S. miltiorrhiza, and purple flower S. miltiorrhiza with different growth ages (one-year-old and two-year-old) were measured, respectively, for analyzing the difference of SPE and contents of specific bioactive constituents between the different growth ages of herbs. Figure 6 displayed the intensity of SPEs in time from the roots of white flower P. grandiflorum (red line) and white flower S. miltiorrhiza (black line) with two years of age as well as the $L$. japonica's flowers buds of Jiufeng number 1 before removing the background (BG), which illustrated that the influence of the external light source on the samples has been eliminated. And Figures 7-10 showed the average SPE intensity (after the subtraction of BG) and average contents of specific bioactive constituents of samples with different growth ages. The total BGs were measured as $5 \pm 1$ counts $/ 0.1 \mathrm{~s}$, and the significant discrepancies between the BGs and all the samples were observed $(P<0.001)$.

As shown in Figure 7, the SPEs intensities of two-yearold $P$. grandiflorum were significantly higher than those of the one-year-old samples in two species $(P<0.05)$. And the contents of total saponins which associated with the quality of $P$. grandiflorum shown in Figure 8 illustrated that one-year-old P. grandiflorum only contained less than $65 \%$ of total saponins of two-year-old one and the contents of total saponins of one-year-old $P$. grandiflorum were about $20 \%$ lower than the provisions of "Chinese Pharmacopoeia."
Therefore, one-year-old $P$. grandiflorum was labeled bad quality herbs. Figure 9 displayed that the SPE intensity of twoyear-old purple flower S. miltiorrhiza was significantly higher than that of the one-year-old sample; however, this result was contrary to the white flower S. miltiorrhiza: the SPE intensity of two-year-old white flower S. miltiorrhiza was lower than that of the one-year-old sample. Interestingly, the data in Figure 10 showed that the contents of tanshinone IIA and salvianolic acid B in two-year-old purple flower S. miltiorrhiza were significantly higher than those of one-year-old sample, while the contents of those two active constituents in twoyear-old white flower S. miltiorrhiza were lower than those of one-year-old sample. These results indicated that the growth age of herbal roots could affect their SPE intensity and there was a close relationship between the bioactive constituents of fresh herbs and their SPE intensities. Besides, the high deviation of SPE intensities among samples suggested that SPE was extremely sensitive and varied in a wide range for slight difference in different plants.

3.2. The Difference of SPEs and Contents of Specific Bioactive Constituents of the Samples in Different Varieties. The data of SPE from the roots with different growth ages of P. grandiflorum and $S$. miltiorrhiza revealed that some interesting difference of SPE existed in different varieties. The data of (SPE-BG)/S from the two-year-old roots of different varieties of $P$. grandiflorum and S. miltiorrhiza were obtained by averaging 30 independent experiments, and then the contents of specific bioactive constituents of different samples were detected. The results were displayed in Figures 11 and 12.

The results suggested that SPEs intensities and contents of specific bioactive constituents from two-year-old roots of different plants varieties showed significant differences $(P<0.05)$. And the change tendencies of SPE intensity and the contents of specific bioactive constituents between different varieties of $P$. grandiflorum and S. miltiorrhiza were the same. These results indicated that the contents of specific active compounds from different varieties herbs were different and there was a close relation between the varieties of plants and their SPE. In order to demonstrate this phenomenon further, the SPEs and the contents of galuteolin and chlorogenic acid of flowers buds from different varieties of L. japonica (four seasons of L. japonica and Jiufeng number 1 as shown in Figure 3) were measured with the measurement procedures. Subsequently, the paired $t$-test was made and the SPE intensities of 20-portion samples in both varieties were averaged in mean \pm SD. The outcomes were displayed in Figures 13 and 14. Notably, all of the flowers buds were in the same growth states.

The result in Figure 13 exhibited that the average intensity of SPE from Jiufeng number 1 was higher than that from four seasons of L. japonica. In Figure 14, the content of chlorogenic acid in Jiufeng number 1 was significantly higher than that from the four seasons of L. japonica, though the content of galuteolin in these two varieties of L. japonica was the same. This result confirmed again that the herbal varieties could affect their SPE intensity and there was a close relation between the bioactive constituents of fresh herbs and their SPE. 


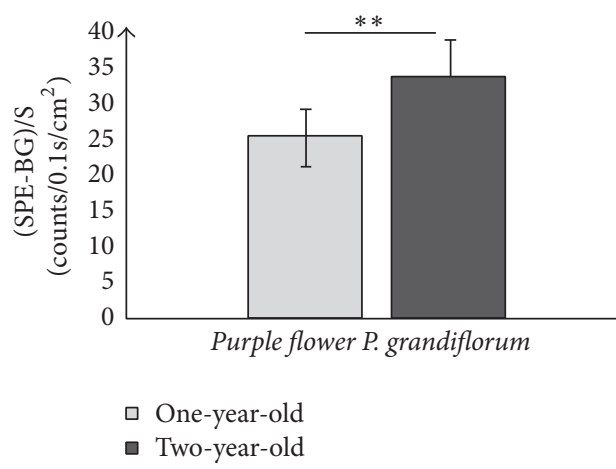

(a)

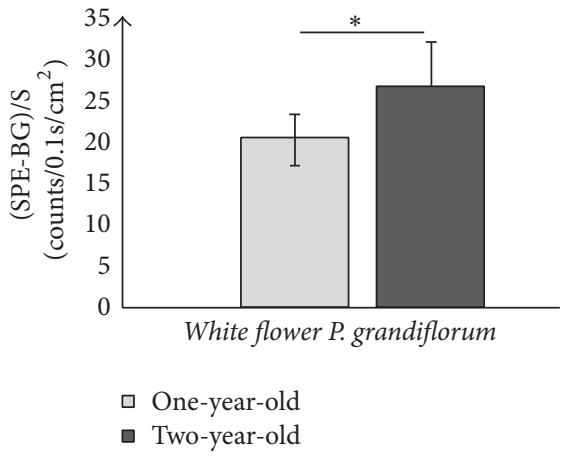

(b)

FIGURE 7: Comparisons of SPEs between different growth ages of roots of purple flower P. grandiflorum (a) and white flower P. grandiflorum (b). ${ }^{*} P<0.05 ;{ }^{* *} P<0.01 . P$ values were obtained by paired $t$-test.

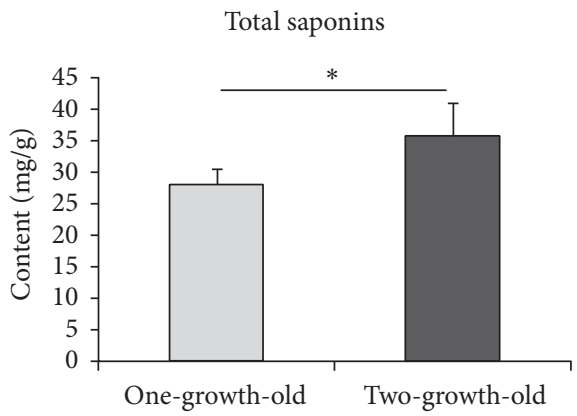

(a)

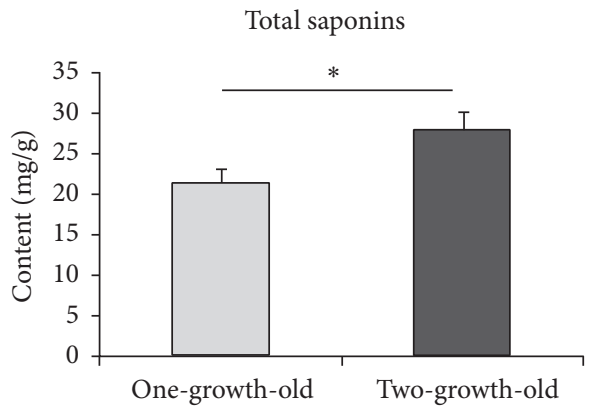

(b)

FIGURE 8: Comparisons of contents of total saponins between different growth ages of roots of purple flower P. grandiflorum (a) and white flower P. grandiflorum (b). ${ }^{*} P<0.05$ (two-tailed, paired $t$-test).

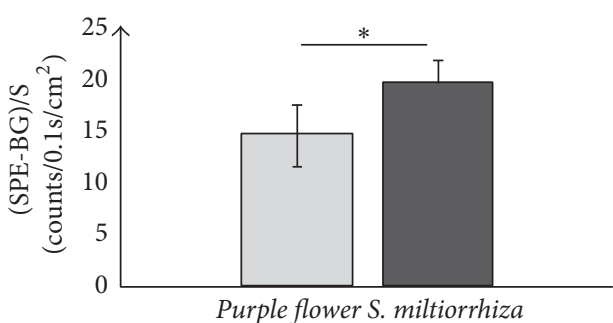

$\square$ One-year-old

- Two-year-old

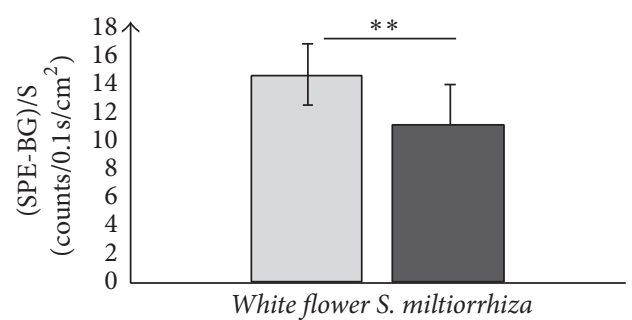

$\square$ One-year-old

- Two-year-old

(a)

(b)

FIGURE 9: Comparisons of SPEs between different growth ages of roots of purple flower S. miltiorrhiza (a) and white flower S. miltiorrhiza (b). ${ }^{*} P<0.05 ;{ }^{* *} P<0.01$. $P$ values were obtained by paired $t$-test.

\section{Discussion}

SPE is an intrinsic property of biological systems. Present studies have suggested that it originates from the relaxation of electronically excited varieties formed in the biological systems during the normal or abnormal oxidative metabolic process $[43,44]$. The production of reactive oxygen varieties (ROS: $\mathrm{O}_{2}{ }^{-}, \mathrm{HO}^{\bullet}, \mathrm{H}_{2} \mathrm{O}_{2},{ }^{1} \mathrm{O}_{2}$ ) in metabolic process plays a key role in the photon emission $[45,46]$. In plants, generation of ROS is related to the enzymatic reactions in mitochondria, chloroplast, and cytoplasm, and the cellular respiration in mitochondria is a main source $[42,47]$. It is stated that ROS with a high positive redox potential has an bility to oxidize various cellular biomolecules such as lipids, proteins and nucleic acids, and then it initiates cascade reactions which are accompanied with the generation of electronically 


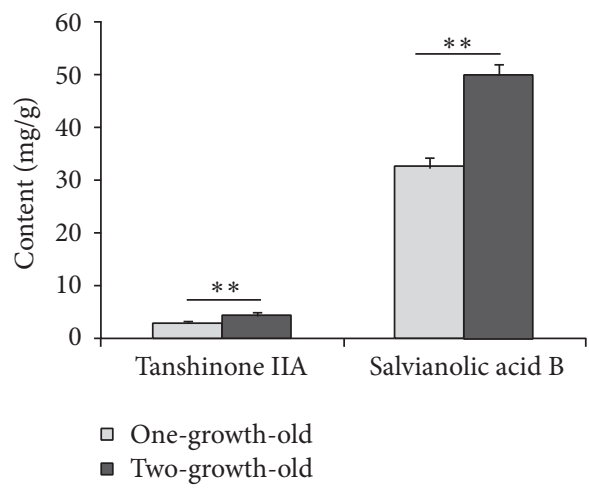

(a)

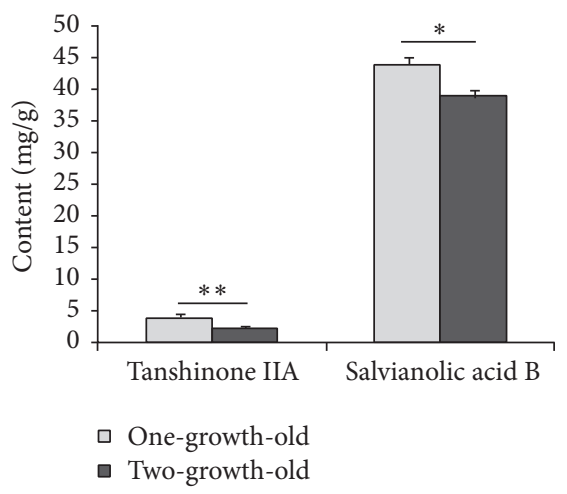

(b)

FIGURE 10: Comparisons of contents of tanshinone IIA and salvianolic acid B between different growth ages of roots of purple flower S. miltiorrhiza (a) and white flower S. miltiorrhiza (b). ${ }^{*} P<0.05 ;{ }^{* *} P<0.01$. $P$ values were obtained by paired $t$-test.

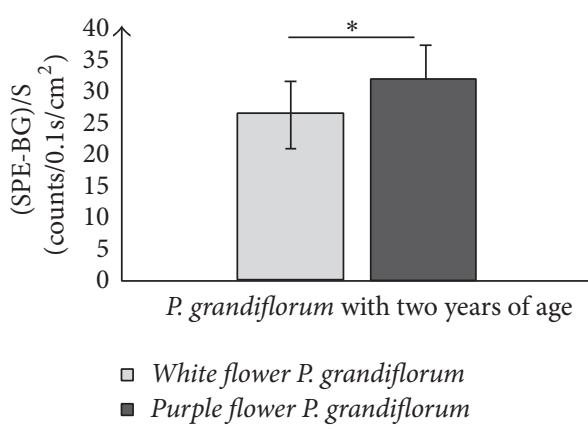

(a)

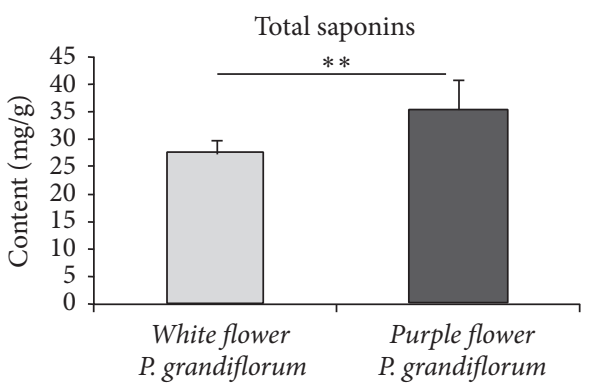

(b)

FIGURE 11: Comparisons of SPEs (a) and contents of total saponins (b) between different varieties of two-year-old roots of P. grandiflorum. ${ }^{*} P<0.05 ;{ }^{* *} P<0.01 . P$ values were obtained by paired $t$-test.

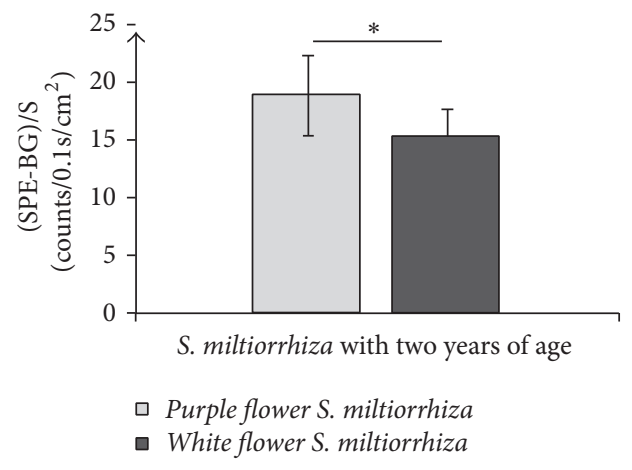

(a)

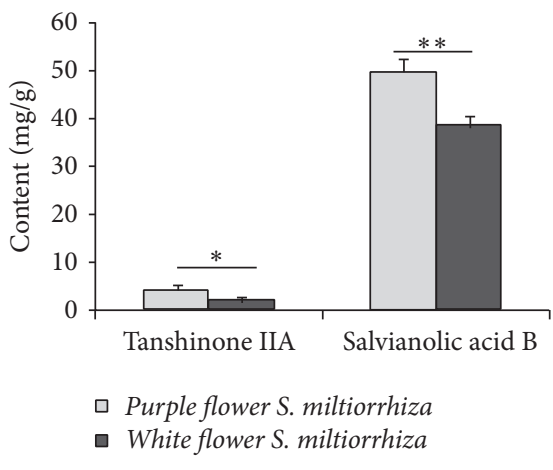

(b)

FiguRE 12: Comparisons of SPEs (a) and contents of tanshinone IIA and salvianolic acid B (b) between different varieties of two-year-old roots of $S$. miltiorrhiza. ${ }^{*} P<0.05 ;{ }^{* *} P<0.01$. $P$ values were obtained by paired $t$-test.

excited varieties such as triplet excited carbonyls $\left({ }^{3} \mathrm{R}=\mathrm{O}^{*}\right)$, excited pigment $\left(P^{*}\right)$, and singlet oxygen $\left({ }^{1} \mathrm{O}_{2}\right)$, resulting in photons emission $[4,43,48-50]$. Thus, the intensity of photon emission is the reflection of underlying metabolic processes in cells.
It is known that the oxidative metabolic processes are the fundamental chemical reactions in the biological system and they reflect the physiological conditions of their system. Therefore, the applications of SPE for the detection of metabolic processes in biological system are widely used. 


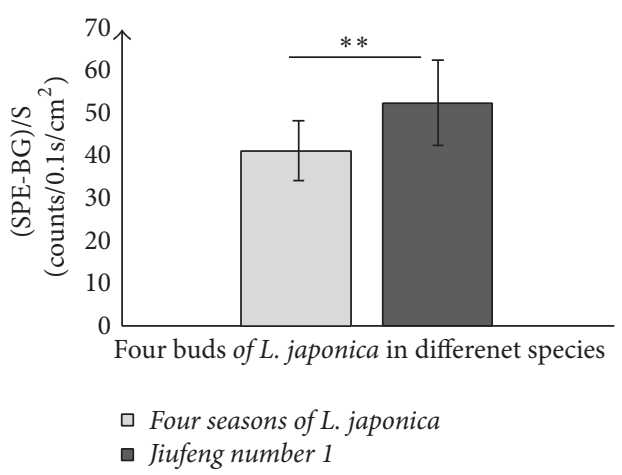

FIGURE 13: Comparisons of SPEs between different varieties of flowers buds for L. japonica. ${ }^{* *} P<0.01 . P$ values were obtained by paired $t$-test.

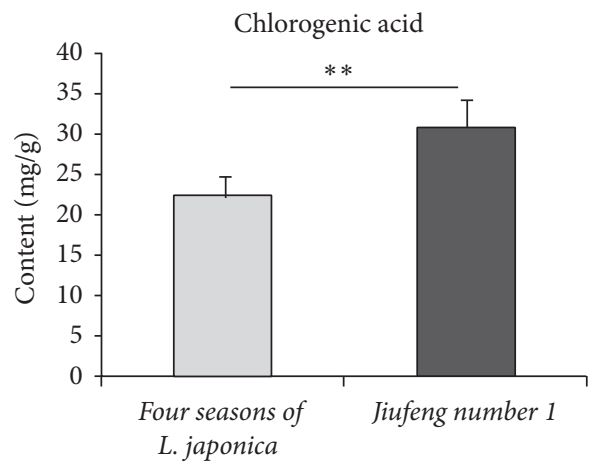

(a)

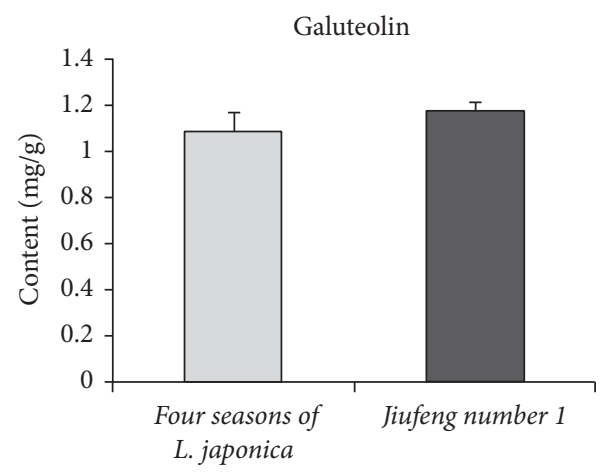

(b)

FiguRE 14: Comparisons of the contents of chlorogenic acid (a) and galuteolin (b) between different varieties of flowers buds for L. japonica. ${ }^{* *} P<0.01$. $P$ values were obtained by paired $t$-test.

Present studies have suggested that the biophoton emission is a meaningful indicator of physiological state in biological systems [8-16, 16-20, 51-53]. In this view, we measured the SPE and contents of specific active compounds of roots and flowers buds with different growth ages and varieties of several fresh Chinese herbal medicines in order to prove whether the growth ages and varieties of fresh herbs could affect their SPE intensity and whether there was a close relationship between the bioactive constituents of fresh herbs and their SPE intensities.

Our data showed that the herbs with different growth age and variety displayed different SPE intensity and content of specific active compounds. In addition, the SPE intensity might have some relationship with the content of specific active compounds of herbs. This may be because the number and types of genes and proteins in different growth ages and varieties of plants are different from each other and these result in varying concentration of specific active compounds. As a consequence, the rate and type of metabolism will also be different, leading to the difference of SPE. Hence, the SPE of a fresh herb is related to its growth age and variety and it was partially correlated to the content of specific active compounds in it at the same time, which reflects the quality and curative effect of herbs. However, further validation is required. Thus, by further research, the SPE of fresh herbs may be a useful index to reflect their growth ages and varieties noninvasively in the future.

It is worth noting that, before we apply this technique practically, we need a large amount of systematic and standard detection for the herbs with different growth ages and varieties using the SPE detection technique. Firstly, these herbs are collected from the field in the way of random sampling and identified by the experts. And then a mutilevel database was found corresponding to the relationship between the various growth ages/varieties of herbs and the characteristics of SPE. Subsequently, the database was optimised according to the actual need. Finally, a feasible "standard" was set up. Once the "standard" was set up, the advantages of the SPE technique can be sufficiently exerted

\section{Conclusion}

In this paper, we reported the changes of SPE intensities and contents of specific active compounds along with growth ages and varieties of several fresh Chinese herbal medicines. Our data showed the statistically significant differences in the photon emission intensities and the contents of specific active compounds between different growth ages and between different varieties of herbs. Although the precise mechanism of SPE has not been fully clarified, these results indicated 
that the contents of specific active compounds of fresh herbs with different growth ages and varieties were significantly different, and this difference could be reflected by their SPE. Therefore, the SPE may be an effective indicator for discriminating herbs with different growth ages and different varieties which greatly affect the quality of Chinese herbs. In this sense, by continuous measurement of SPE from many more types of herbs with different growth ages, varieties, harvest seasons, authenticity, and so forth, this novel and noninvasive method may make a great contribution to solving the tough problems existing in the quality screening of fresh Chinese herbal medicine in certain degree in the future.

\section{Competing Interests}

The authors declare that they have no competing interests.

\section{Acknowledgments}

This work is funded by grants from the National Natural Science Foundation of China (no. 81273997), Ministry of Science and Technology of China (2014DFA30380), and Shandong Provincial Natural Science Foundation, China (ZR2015HQ016). The authors thank all the individuals who participated in these studies and all the researchers, technicians, and administrative staff who have enabled this work. They would like to acknowledge Dr. Eduard Van Wijk (Meluna Research), Mei Wang, and Dr. Yu Yan for supervising the construction of the equipment and technical assistance.

\section{References}

[1] F.-A. Popp, "Properties of biophotons and their theoretical implications," Indian Journal of Experimental Biology, vol. 41, no. 5, pp. 391-402, 2003.

[2] M. Cifra and P. Pospíšil, "Ultra-weak photon emission from biological samples: definition, mechanisms, properties, detection and applications," Journal of Photochemistry and Photobiology B: Biology, vol. 139, pp. 2-10, 2014.

[3] A. Prasad and P. Pospíšil, "Towards the two-dimensional imaging of spontaneous ultra-weak photon emission from microbial, plant and animal cells," Scientific Reports, vol. 3, article 1211, 2013.

[4] A. Rastogi and P. Pospíšl, "Spontaneous ultraweak photon emission imaging of oxidative metabolic processes in human skin: effect of molecular oxygen and antioxidant defense system," Journal of Biomedical Optics, vol. 16, no. 9, Article ID 096005, 2011.

[5] H. Inagaki, T. Imaizumi, G.-X. Wang et al., "Spontaneous ultraweak photon emission from rice (Oryza sativa L.) and paddy weeds treated with a sulfonylurea herbicide," Pesticide Biochemistry and Physiology, vol. 89, no. 2, pp. 158-162, 2007.

[6] Y. Tian, C. Yang, and H. Xu, "Insect spontaneous ultraweak photon emission as an indicator of insecticidal compounds," Journal of Photochemistry and Photobiology B: Biology, vol. 140, pp. 79-84, 2014.

[7] K. Kato, H. Iyozumi, C. Kageyama, H. Inagaki, A. Yamaguchi, and H. Nukui, "Application of ultra-weak photon emission measurements in agriculture," Journal of Photochemistry and Photobiology B: Biology, vol. 139, pp. 54-62, 2014.
[8] T. Hakamata, K. Kato, T. Makino, and S. Yamamoto, "Weak photon emission from the Japanese black pine inoculated with pine wood nematode," Japanese Journal of Phytopathology, vol. 70, no. 3, pp. 162-167, 2004.

[9] T. Makino, K. Kato, H. Iyozumi, H. Honzawa, Y. Tachiiri, and M. Hiramatsu, "Ultraweak luminescence generated by sweet potato and Fusarium oxysporum interactions associated with a defense response," Photochemistry and Photobiology, vol. 64, no. 6, pp. 953-956, 1996.

[10] F. Musumeci, L. A. Applegate, G. Privitera, A. Scordino, S. Tudisco, and H. J. Niggli, "Spectral analysis of laser-induced ultraweak delayed luminescence in cultured normal and tumor human cells: temperature dependence," Journal of Photochemistry and Photobiology B: Biology, vol. 79, no. 2, pp. 93-99, 2005.

[11] K. Nakamura and M. Hiramatsu, "Ultra-weak photon emission from human hand: influence of temperature and oxygen concentration on emission," Journal of Photochemistry and Photobiology B: Biology, vol. 80, no. 2, pp. 156-160, 2005.

[12] A. Rastogi and P. Pospíšil, "Ultra-weak photon emission as a non-invasive tool for the measurement of oxidative stress induced by UVA radiation in Arabidopsis thaliana," Journal of Photochemistry and Photobiology B: Biology, vol. 123, pp. 59-64, 2013.

[13] E. Bertogna, J. Bezerra, E. Conforti, and C. M. Gallep, "Acute stress in seedlings detected by ultra-weak photon emission," Journal of Photochemistry and Photobiology B: Biology, vol. 118, no. 1, pp. 74-76, 2013.

[14] W. L. Chen, D. Xing, S. Tan, Y. Tang, and Y. He, "Imaging of ultra-weak bio-chemiluminescence and singlet oxygen generation in germinating soybean in response to wounding," Luminescence, vol. 18, no. 1, pp. 37-41, 2003.

[15] R. Kawabata, T. Miike, M. Uefune, H. Okabe, M. Takagi, and S. Kai, "Biophoton measurement of herbivore-induced plant responses," Japanese Journal of Applied Entomology and Zoology, vol. 48, no. 4, pp. 289-296, 2004.

[16] H. Iyozumi, K. Kato, C. Kageyama et al., "Plant defense activators potentiate the generation of elicitor-responsive photon emission in rice," Physiological and Molecular Plant Pathology, vol. 66, no. 1-2, pp. 68-74, 2005.

[17] F. Musumeci, G. Privitera, A. Scordino et al., "Discrimination between normal and cancer cells by using spectral analysis of delayed luminescence," Applied Physics Letters, vol. 86, no. 15, Article ID 153902, 2005.

[18] H.-W. Kim, S.-B. Sim, C.-K. Kim et al., "Spontaneous photon emission and delayed luminescence of two types of human lung cancer tissues: adenocarcinoma and Squamous cell carcinoma," Cancer Letters, vol. 229, no. 2, pp. 283-289, 2005.

[19] M. Takeda, M. Kobayashi, M. Takayama et al., "Biophoton detection as a novel technique for cancer imaging," Cancer Science, vol. 95, no. 8, pp. 656-661, 2004.

[20] M. Iranifam, "Analytical applications of chemiluminescence methods for cancer detection and therapy," TrAC-Trends in Analytical Chemistry, vol. 59, pp. 156-183, 2014.

[21] M. Yang, J. Pang, J. Liu, Y. Liu, H. Fan, and J. Han, "Spectral discrimination between healthy people and cold patients using spontaneous photon emission," Biomedical Optics Express, vol. 6, no. 4, pp. 1331-1339, 2015.

[22] L. Lanzanò, A. Scordino, S. Privitera, S. Tudisco, and F. Musumeci, "Spectral analysis of delayed luminescence from human skin as a possible non-invasive diagnostic tool," European Biophysics Journal, vol. 36, no. 7, pp. 823-829, 2007. 
[23] E. Van Wijk, M. Kobayashi, R. Van Wijk, and J. Van Der Greef, "Imaging of ultra-weak photon emission in a rheumatoid arthritis mouse model," PLoS ONE, vol. 8, no. 12, Article ID e84579, 2013.

[24] P. Chen, L. Zhang, F. Zhang et al., "Spectral discrimination between normal and leukemic human sera using delayed luminescence," Biomedical Optics Express, vol. 3, no. 8, pp. 17871792, 2012.

[25] X. L. Zhao, J. X. Pang, J. L. Fu et al., "Spontaneous photon emission: a promising non-invasive diagnostic tool for breast cancer," Journal of Photochemistry and Photobiology B: Biology, vol. 166, pp. 232-238, 2017.

[26] J. A. Ives, E. P. A. V. Wijk, N. Bat et al., "Ultraweak photon emission as a non-invasive health assessment: a systematic review," PLoS ONE, vol. 9, no. 2, Article ID e87401, 2014.

[27] M. Hossu, L. Ma, and W. Chen, "Nonlinear enhancement of spontaneous biophoton emission of sweet potato by silver nanoparticles," Journal of Photochemistry and Photobiology B: Biology, vol. 99, no. 1, pp. 44-48, 2010.

[28] R. R. Chaudhury, "Herbal remedies and traditional medicines in reproductive health care practices and their clinical evaluation," Journal of Reproductive Health \& Medicine, vol. 1, no. 1, pp. 44-46, 2015.

[29] O. Gureje, G. Nortje, V. Makanjuola, B. D. Oladeji, S. Seedat, and R. Jenkins, "The role of global traditional and complementary systems of medicine in the treatment of mental health disorders," The Lancet Psychiatry, vol. 2, no. 2, pp. 168-177, 2015.

[30] J.-Y. Liu, X.-X. Chen, S. C.-W. Tang et al., "Edible plants from traditional Chinese medicine is a promising alternative for the management of diabetic nephropathy," Journal of Functional Foods, vol. 14, pp. 12-22, 2015.

[31] C. X. Hu and G. W. Xu, "Metabolomics and traditional Chinese medicine," TrAC Trends in Analytical Chemistry, vol. 61, pp. 207-214, 2014.

[32] Y. Jiang, B. David, P. Tu, and Y. Barbin, "Recent analytical approaches in quality control of traditional Chinese medicines-a review," Analytica Chimica Acta, vol. 657, no. 1, pp. 9-18, 2010.

[33] Z. N. An, "Discussion on the effect of chinese herbal medicine quality factors and solving measures," Journal of Practical Traditional Chinese Internal Medicine, vol. 26, no. 9, pp. 82-83, 2012.

[34] WHO, General Guidelines for Methodologies on Research and Evaluation of Traditional Medicines, World Health Organization, 2000.

[35] J. Wang, R. van der Heijden, S. Spruit et al., "Quality and safety of Chinese herbal medicines guided by a systems biology perspective," Journal of Ethnopharmacology, vol. 126, no. 1, pp. 31-41, 2009.

[36] P.-S. Xie and A. Y. Leung, "Understanding the traditional aspect of Chinese medicine in order to achieve meaningful quality control of Chinese materia medica," Journal of Chromatography A, vol. 1216, no. 11, pp. 1933-1940, 2009.

[37] J. Pang, J. Fu, M. Yang et al., "Correlation between the different therapeutic properties of Chinese medicinal herbs and delayed luminescence," Luminescence, vol. 31, no. 2, pp. 323-327, 2016.

[38] M. Sun, L. Li, M. Wang et al., "Effects of growth altitude on chemical constituents and delayed luminescence properties in medicinal rhubarb," Journal of Photochemistry and Photobiology B: Biology, vol. 162, pp. 24-33, 2016.
[39] Z. X. Li, C. D. Xu, and W. B. Han, "Effect of growth ages on quality of P. grandiflorum," China Journal of Chinese Materia Medica, vol. 26, no. 9, pp. 598-599, 2001.

[40] L. Zhou, W. Yi, J. Qi, P. Sun, and X. Li, "Effect of varieties and growth ages on root yield and bioactive components accumulation dynamics of $S$. miltiorrhizae," Chinese Wild Plant Resources, vol. 31, no. 5, pp. 8-17, 2012.

[41] L. Feng, H. Ji, D. Wang, J. Wang, and R. Lu, "Comparison of the volatile oil from S. miltiorrhiza bunge. F. alba. and S. miltiorrhiza bunge," Fine Chemicals, vol. 26, no. 7, pp. 662-670, 2009.

[42] X. Ren, Y. Xie, Y. Zhang, Y. Dong, and H. Guo, "Analysis of clinical application of L. japonica," West China Medical Journal, vol. 21, no. 7, pp. 1175-1176, 2009.

[43] R. Van Wijk, E. P. A. Van Wijk, F. A. C. Wiegant, and J. Ives, "Free radicals and low-level photon emission in human pathogenesis: state of the art," Indian Journal of Experimental Biology, vol. 46, no. 5, pp. 273-309, 2008.

[44] J. Sławinski, "Biophotons from stressed and dying organisms: toxicological aspects," Indian Journal of Experimental Biology, vol. 41, no. 5, pp. 483-493, 2003.

[45] P. Pospíšil, A. Prasad, and M. Rác, "Role of reactive oxygen species in ultra-weak photon emission in biological systems," Journal of Photochemistry and Photobiology B: Biology, vol. 139, pp. 11-23, 2014.

[46] A. Rastogi and P. Pospíšil, "Effect of exogenous hydrogen peroxide on biophoton emission from radish root cells," Plant Physiology and Biochemistry, vol. 48, no. 2-3, pp. 117-123, 2010.

[47] M. M. Sedensky and P. G. Morgan, "Mitochondrial respiration and reactive oxygen species in C. elegans," Experimental Gerontology, vol. 41, no. 10, pp. 957-967, 2006.

[48] S. Miyamoto, G. E. Ronsein, F. M. Prado et al., "Biological hydroperoxides and singlet molecular oxygen generation," IUBMB Life, vol. 59, no. 4-5, pp. 322-331, 2007.

[49] G. F. Fedorova, A. V. Trofimov, R. F. Vasilev, and T. L. Veprintsev, "Peroxy-radical-mediated chemiluminescence: mechanistic diversity and fundamentals for antioxidant assay," ARKIVOC: Archive for Organic Chemistry, vol. 38, no. 8, pp. 163-215, 2007.

[50] S. Birtic, B. Ksas, B. Genty, M. J. Mueller, C. Triantaphylidès, and M. Havaux, "Using spontaneous photon emission to image lipid oxidation patterns in plant tissues," Plant Journal, vol. 67, no. 6, pp. 1103-1115, 2011.

[51] X. Zhao and J. Han, "The connotation of the Quantum Traditional Chinese Medicine and the exploration of its experimental technology system for diagnosis," Drug Discoveries and Therapeutics, vol. 7, no. 6, pp. 225-232, 2013.

[52] J. Pang, M. Yang, J. Fu et al., "Classification of Chinese herbs based on the cluster analysis of delayed luminescence," Luminescence, vol. 31, no. 2, pp. 491-498, 2016.

[53] Y. Gabe, O. Osanai, and Y. Takema, "The relationship between skin aging and steady state ultraweak photon emission as an indicator of skin oxidative stress in vivo," Skin Research and Technology, vol. 20, no. 3, pp. 315-321, 2014. 


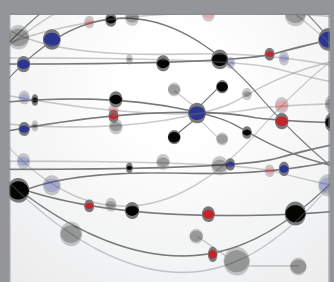

The Scientific World Journal
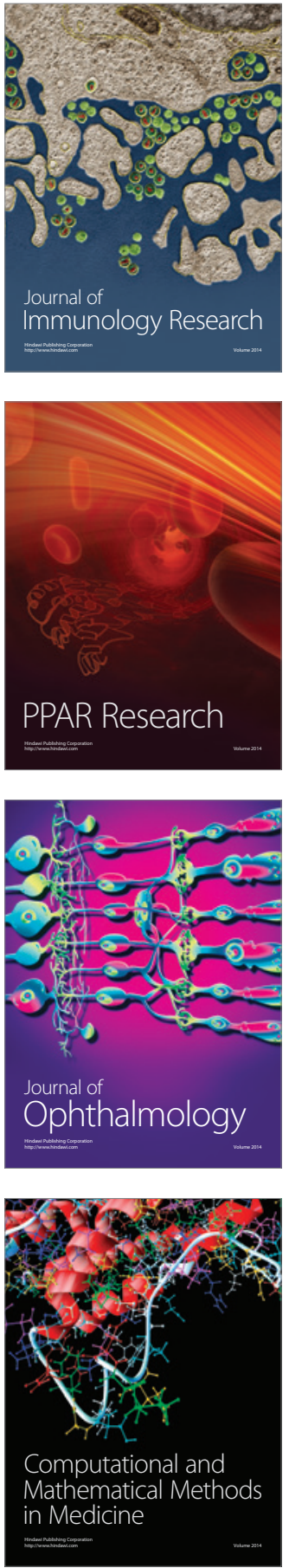

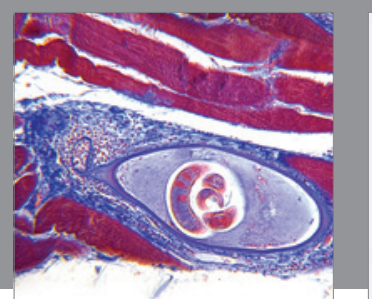

Gastroenterology Research and Practice
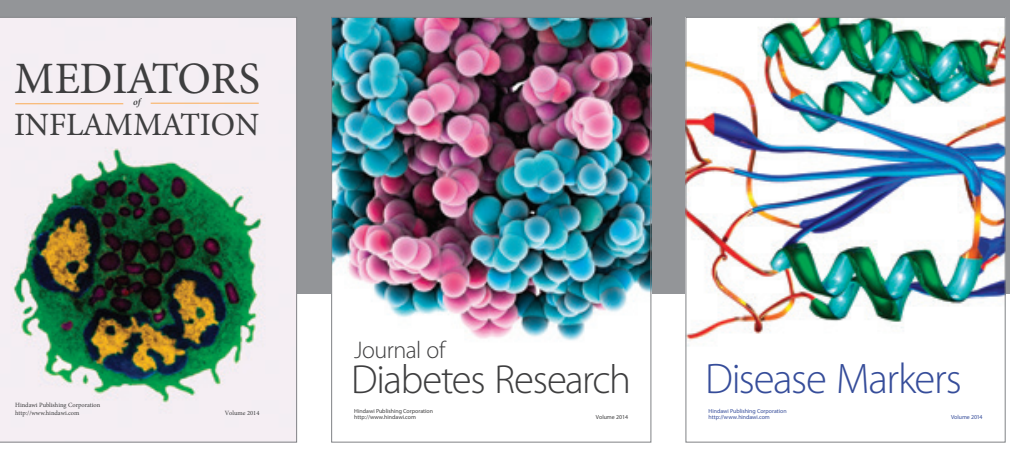

Disease Markers

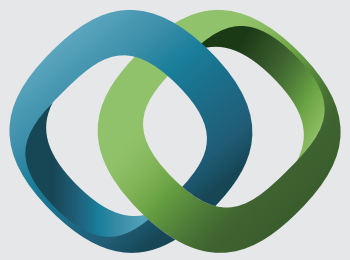

\section{Hindawi}

Submit your manuscripts at

https://www.hindawi.com
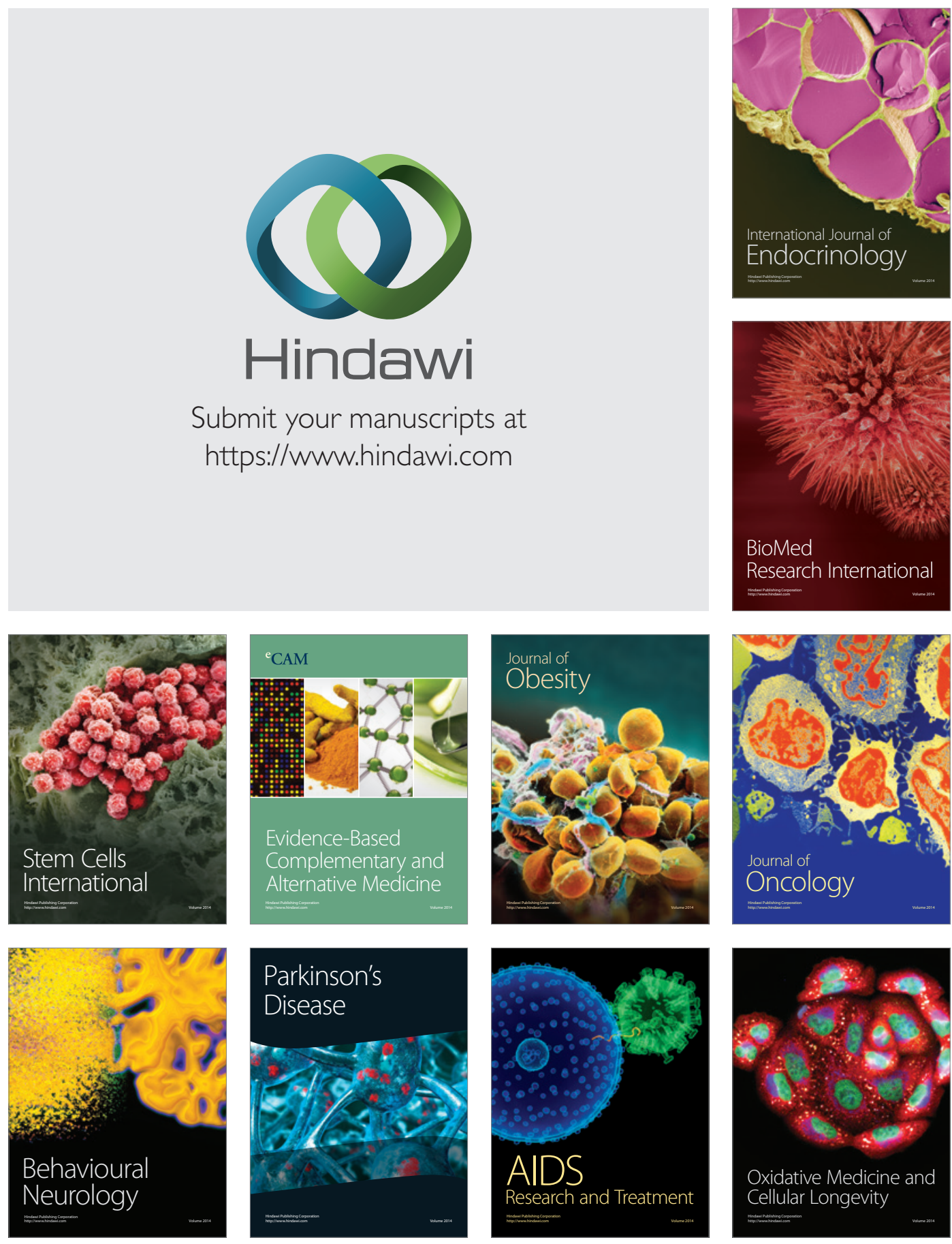